\title{
Resumo
}

\section{Estudo MONISA: características e aspectos metodológicos}

\section{MONISA study: characteristics and methodological aspects}

Thiago Ferreira de Sousa'

Silvio Aparecido Fonseca',",

Helma Pio Mororó José"l'

\section{Markus Vinicius Nahas'v}

'Grupo de Pesquisa em Atividade Física e Saúde da Universidade Estadual de Santa Cruz, Ilhéus, Bahia.

"Universidade Estadual de Santa Cruz, Ilhéus, Bahia.

I"Universidade Estadual do Sudoeste da Bahia, Vitória da Conquista, Bahia.

vUniversidade Federal de Santa Catarina, Florianópolis, Santa Catarina.

Correspondência: Thiago Ferreira de Sousa. Universidade Estadual de Santa Cruz, Departamento de Ciências da Saúde, Grupo de Pesquisa em Atividade Física e Saúde. Campus Soane Nazaré de Andrade, km 16, Rodovia Ilhéus-Itabuna, Ilhéus, BA CEP 45662-900. E-mail: tfsousa_thiago@yahoo.com.br. Fone: +55 (73) 3680-5116

O objetivo é apresentar as características e os procedimentos metodológicos adotados no Estudo Monitoramento dos Indicadores de Saúde e Qualidade de Vida de Acadêmicos (MONISA). Trata-se de um estudo prospectivo, do tipo painel, com inquéritos bianuais em amostras representativas de estudantes de uma universidade pública do Estado da Bahia. Este estudo realizará cinco inquéritos, perfazendo 10 anos de monitoramento (2010 a 2018). A amostra é estratificada e proporcional aos cursos de graduação presenciais, período de estudo (noturno e diurno) e ano de ingresso na universidade. Por fim, os estudantes universitários são selecionados em cada estrato, por meio da lista de matrícula em ordem alfabética. Para obtenção das informações é utilizado um questionário estruturado, com as seguintes seções: indicadores sociodemográficos; indicadores do estilo de vida e saúde; hábitos alimentares e controle do peso corporal; atividades físicas e opções de lazer; comportamentos preventivos; e, indicadores do ambiente e condições de aprendizagem. O pioneirismo do Estudo MONISA poderá auxiliar no esclarecimento de possíveis tendências relacionadas à saúde de estudantes universitários brasileiros e subsidiar informações para propor programas ou projetos de promoção da saúde e qualidade de vida em nível organizacional local.

Palavras-chave: Estudantes. Estudos longitudinais. Estudos transversais. Amostragem aleatória e sistemática. Conduta de saúde. Qualidade de vida. 


\section{Abstract}

The objective is to present the characteristics and methodological procedures adopted in the MONISA Study (Surveillance of health and quality of life indicators of college students). This is a prospective, panel type study, with biannual surveys of representative samples of undergraduate students at a public university in the State of Bahia, Brazil. This study carried out five surveys, totaling 10 years of monitoring (2010-2018). The sample is stratified and proportional to the courses, study period (day and night) and year of attending university. Finally, college students are selected at each stratum, through the registration list in alphabetical order. To obtain the information is used a structured questionnaire with the following sections: socio-demographic indicators, indicators of lifestyle and health, eating habits and body weight control, physical activity and other leisure options, preventive behaviors, and indicators environment and learning conditions. The novel aspects of this study may help in clarifying possible trends related to the health of Brazilian college students and to subsidize policies and programs or projects to promote health and quality of life in local, organizational level.

Keywords: Students. Longitudinal studies. Cross-sectional studies. Random and systematic sampling. Health behavior. Quality of life.

\section{Introdução}

$\mathrm{O}$ crescimento de mortes relacionadas às doenças crônicas não transmissíveis (DCNT) tem sido observado em diversos países ${ }^{1}$ e diferentes fatores de risco, como a hipertensão arterial, tabagismo, glicemia sanguínea elevada, inatividade física, sobrepeso e obesidade são associados às DCNT ${ }^{1}$. Diante dos potenciais malefícios dos fatores de risco à saúde da população e às despesas dos sistemas de saúde, diversos países conduzem estudos de monitoramento, incluindo o Brasil a partir do sistema de vigilância por inquérito telefônico (VIGITEL) ${ }^{2}$, bem como outros inquéritos com populações específicas, como trabalhadores de universidades brasileiras (Pró-Saúde e ELSA) ${ }^{3,4}$.

Tais iniciativas são importantes devido às peculiaridades, determinantes e condicionantes da saúde de pessoas que vivem em ambientes específicos, incluindo a crescente população de estudantes no ensino superior. Contudo, estudos de monitoramento para estudantes universitários ainda não parecem acompanhar iniciativas observadas em outros grupos populacionais. Em função das lacunas apontadas, este artigo visa apresentar as características e os procedimentos metodológicos adotados no Estudo MONISA (Monitoramento dos Indicadores de Saúde e Qualidade de Vida de Acadêmicos).

\section{Características e aspectos metodológicos do Estudo MONISA}

O Estudo MONISA é caracterizado como prospectivo, do tipo painel, pois serão realizadas coletas bianuais com amostras representativas de estudantes de uma universidade pública da região sul do Estado da Bahia. Este estudo tem como objetivo monitorar e caracterizar os indicadores do estilo de vida e as condições ambientais e de aprendizagem em estudantes por um período de dez anos (2010-2018). O desenho da investigação foi aprovado pelo comitê de ética em pesquisa local ( $\left.n^{\circ} 382 / 10\right)$.

A população inclui os estudantes 
matriculados no segundo semestre letivo dos anos de inquérito em todos os cursos de graduação presenciais. São excluídos os estudantes de ensino à distância, portadores de diploma de ensino superior matriculados em disciplinas da instituição e estudantes aprovados para o ingresso no segundo semestre. Para os cálculos amostrais são considerados: população de referência, nível de confiança de 95\%, erro tolerável de amostragem de três pontos percentuais e uma prevalência de $50 \%$, acrescidos $20 \%$ para possíveis perdas e $15 \%$ para estudos de associação. Os sujeitos selecionados devem ser procurados em até três tentativas; caso não encontrados ou não tenham interesse em participar são considerados como perdas e recusas, respectivamente, não havendo reposição para ambas as situações.

A amostra é estratificada e proporcional aos cursos da instituição, período de estudo (noturno e diurno) e ano de ingresso na universidade (considerando quatro categorias, tendo em vista que a maioria dos cursos é de no mínimo quatro anos para a conclusão). Em cada estrato, os estudantes são selecionados aleatoriamente por meio da lista de matrícula em ordem alfabética. O fluxograma do Estudo MONISA é apresentado na Figura 1.

Nos meses de julho e agosto são realizados os treinamentos com a equipe de coleta de dados e as coletas realizadas nas dependências da instituição, nos meses de setembro a novembro. As informações são obtidas por meio do questionário Isaq-A (Indicadores de Saúde e Qualidade de Vida de Acadêmicos $)^{5}$ que foi construído com base as perguntas na forma original ou com pequenas adaptações de instrumentos para adultos e estudantes universitários. O Isaq-A foi previamente testado em relação à validade de face e conteúdo; clareza e pré-testagem; e reprodutibilidade com intervalo de uma semana, apresentando indicadores satisfatórios para a sua aplicação. Sua versão final é composta por 71 perguntas, a ser autopreenchido em sala de aula, e suas seções são: informações sobre o curso; indicadores sociodemográficos; indicadores do estilo de vida e saúde; hábitos alimentares e controle do peso corporal; atividades físicas e opções

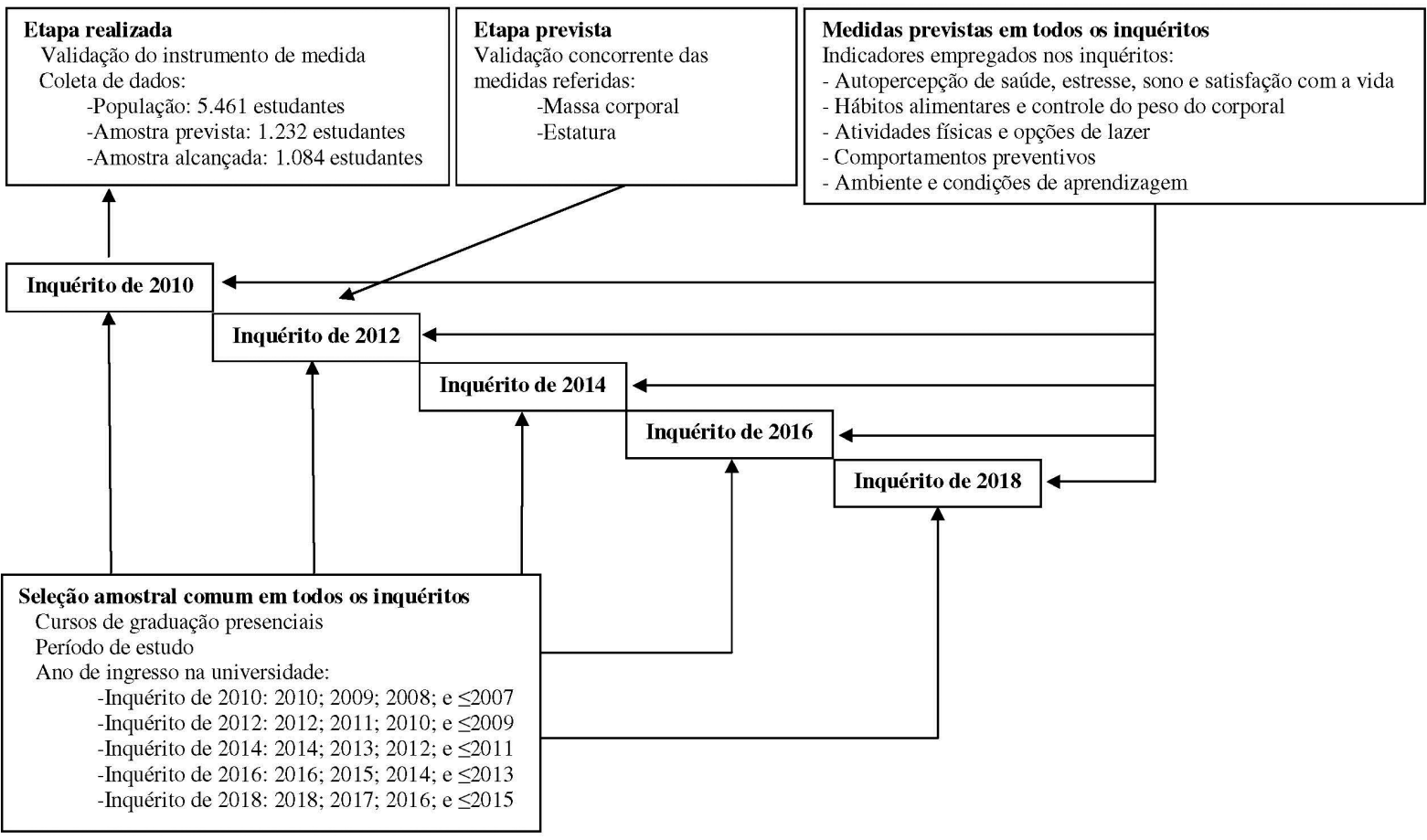

Figura 1 - Fluxograma dos inquéritos do Estudo MONISA.

Figure 1 - Flowchart of surveys in the MONISA Study. 
de lazer; comportamentos preventivos; e indicadores do ambiente e das condições de aprendizagem.

\section{Considerações finais}

O rigor metodológico do Estudo MONISA é um dos seus pontos fortes e sua logística de fácil aplicação foi observada no primeiro inquérito (baseline) de 2010, que obteve taxa de resposta de $88 \%(\mathrm{n}=1.084)$. As informações do primeiro inquérito foram divulgadas em forma de palestra, jornal da instituição e artigos científicos ${ }^{6-9}$. A vigilância no prosseguimento do Estudo MONISA poderá esclarecer possíveis tendências relacionadas à saúde de estudantes universitários brasileiros e subsidiar informações para propor programas ou projetos de promoção da saúde e qualidade de vida no nível organizacional local. Em caso de implementação de intervenções, potenciais repercussões salutares aos sujeitos em médio e longo prazo são esperadas.

\section{Referências}

1. World Health Organization [WHO]. Global health risks: mortality and burden of disease attributable to selected major risks. Geneva: WHO; 2009.

2. Ministério da Saúde do Brasil. Vigilância de Fatores de Risco e Proteção para Doenças Crônicas por Inquérito Telefônico: estimativas sobre frequência e distribuição sócio-demográfica de fatores de risco e proteção para doenças crônicas nas capitais dos 26 Estados brasileiros e no Distrito Federal em 2008. Brasília: Ministério da Saúde; 2011.

3. Faerstein E, Chor D, Lopes CS, Werneck GL. Estudo PróSaúde: características gerais e aspectos metodológicos. Rev Bras Epidemiol 2005; 8(4): 454-66.

4. Departamento de Ciência e Tecnologia, Secretaria de Ciência, Tecnologia e Insumos Estratégicos, Ministério da Saúde. ELSA Brasil: maior estudo epidemiológico da América Latina. Rev Saúde Pública 2009; 43(1): 1-2.
5. Sousa TF, Fonseca SA, José HPM, Nahas MV. Validade e reprodutibilidade do questionário Indicadores de Saúde e Qualidade de Vida de Acadêmicos (Isaq-A). Arq Cien Esp 2012 (no prelo).

6. Sousa TF, Nahas MV. Prevalência e fatores associados a menores níveis de prática de atividades físicas no lazer em estudantes de uma universidade pública do Estado da Bahia. Rev Bras Ativ Fis Saúde 2011; 16(4): 322-29.

7. Sousa TF. Atividades físicas praticadas no lazer por universitários de uma instituição pública do Nordeste do Brasil. Rev Bras Ativ Fis Saúde 2012; 17(2): 125-31.

8. Sousa TF, José HPM, Barbosa AR. Condutas negativas à saúde em estudantes universitários brasileiros. Ciênc Saúde Colet 2013 (no prelo).

9. Sousa TF, Virtuoso Junior JS, Barbosa AR. Autovaloración de salud: localización de la pregunta en las encuestas epidemiológicas. Gac Sanit 2013 (no prelo).

Recebido em: 04/11/11 Versão final apresentada em: 25/07/12 Aprovado em: 12/09/12 